\title{
OPTICAL MONITORING
}

\section{OF GAMMA-RAY LOUD BLAZARS}

\author{
C.M. RAITERI ${ }^{1}$, G. GHISELLINI ${ }^{1}$, M. VILLATA ${ }^{1}$, \\ G. DE FRANCESCO ${ }^{1}$, S. BOSIO ${ }^{2}$, G. LATINI ${ }^{2}$, G. CHIUMIENTO ${ }^{1}$, \\ L. LANTERI ${ }^{1}$, G. MASSONE ${ }^{1}$, F. RACIOPPI ${ }^{1}$, AND R.L. SMART ${ }^{1}$ \\ 1 Osservatorio Astronomico di Torino \\ Strada Osservatorio 20, I-10025 Pino Torinese (TO), Italy \\ 2 Istituto di Fisica Generale dell'Università \\ Via Pietro Giuria 1, I-10125 Torino, Italy
}

The observations by the Compton Gamma Ray Observatory (CGRO) have shown that highly variable and radio-loud quasars emit a significant fraction of their energy in the $\gamma$ band. According to the Inverse Compton model, the $\gamma$-ray emission is due to upscattering of soft (IR-optical-UV) photons by high energy particles. Optical monitoring is thus of great value in providing information on the mechanisms that rule the production of the seed photons for the $\gamma$-ray radiation and on the $\gamma$-ray emission itself. In particular, detection of variability correlations between optical and $\gamma$-ray emissions would be a crucial test for the theoretical predictions.

For the above reasons, we have started an optical monitoring campaign of $\gamma$-ray loud blazars in Torino (Italy) since November 1994 (Villata et al., 1995). Our list includes 30 objects. In order to have a better temporal coverage, we entered collaborations with other monitoring groups on some sources [e.g. Massaro et al. (1995) on 0422+004; Ghisellini et al. (1995) and Latini et al. (these proceedings) on 0716+714; Sillanpää et al. (1995) and Takalo et al. (these proceedings) on $0851+202$, as part of the OJ-94 Project, where we are involved also for $0219+428$ and $0235+164]$. Data have been taken with the Torino $1.05 \mathrm{~m}$ R.E.O.S.C. telescope equipped with a $1242 \times 1152$ pixels CCD camera and standard Johnson $U B V$ and Cousins $R I$ filters. Observations have been done in the $R$ and $B$ bands.

Most of the sources show noticeable optical variations on both long and short time scales, and for some of them we could detect also intranight 

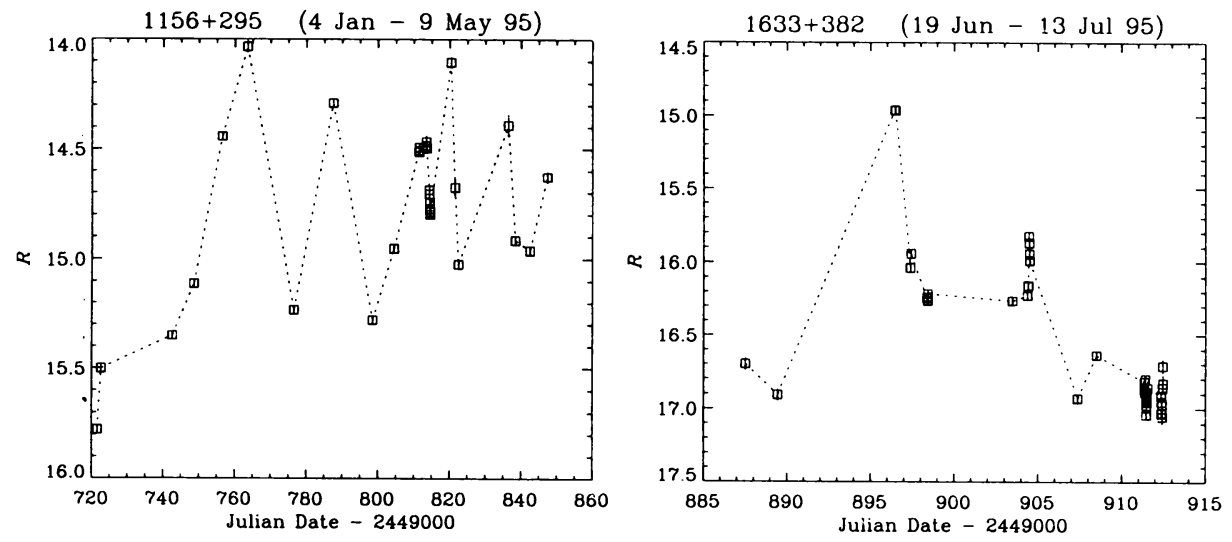

Figure 1. Light curves in the $R$ band for the two quasars $1156+295$ and $1633+382$

variability.

Figure 1 shows part of the light curves in the $R$ band for $1156+295$ and $1633+382$. The source magnitude is given as the average value between the magnitudes obtained with respect to two of the three reference stars that we chose in the source field. The optical emission of the highly polarized quasar $1156+295$ (4C 29.45) has been rapidly and widely changing during all our monitoring period $\left(\Delta R_{\max }=1.75, \Delta B_{\max }=1.81\right)$, with the steepest decrease of about $0.9 \mathrm{mag}(R)$ in two days (JD $\sim 2449821$ ); a peak was detected at JD $=2449836$, during the CGRO observation period (18 Apr - 2 May 95), when a very high $\gamma$-ray emission (maybe nne order of magnitude greater than the previous detection of $6.310^{-7} \mathrm{~cm}^{-2} \mathrm{~s}^{-1}$ in 1991) was registered. Consistent intranight variability has been found for the quasar $1633+382(4 \mathrm{C} 38.41)$; on JD $=2449904$ a variation $\Delta R=0.34$ mag was detected in half an hour. After a period of quiet emission, this source reached its historical maximum $(R=14.96 \pm 0.03)$ on June 27, 1995 (Bosio et al., 1995); observations in the radio band are strongly encouraged, since a time-delayed correlated peak could be detected.

\section{References}

Bosio, S., De Francesco, G., Ghisellini, G. et al. (1995) 4C 38.41, IAU Circ., no. 6183 Ghisellini, G., Villata, M., Raiteri, C.M. et al. (1995), $A \& A$, (submitted)

Latini, G., Bosio, S., De Francesco, G. et al. (these proceedings) Optical Monitoring of the Gamma-ray Loud BL Lac Object S5 0716+714

Massaro, E., Nesci, R., Trevese, D. et al. (1995), A\&A, (submitted)

Sillanpäă, A., Takalo, L.O., Lehto, H.J. et al. (1995) Confirmation of the 12 Year Optical Outburst Cycle in Blazar OJ 287, $A \& A$, (in press)

Takalo, L.O., Sillanpää, A., Pursimo, T. et al. (these proceedings) Variability Characteristics of Blazar OJ 287

Villata, M., Raiteri, C.M., Ghisellini, G. et al. (1995), A\&AS, (submitted) 